\title{
The boundary conditions of priming of visual search: From passive viewing through task-relevant working memory load
}

\author{
Árni Kristjánsson • Styrmir Saevarsson • Jon Driver
}

Published online: 17 January 2013

(C) Psychonomic Society, Inc. 2013

\begin{abstract}
Priming of visual search has a dominating effect upon attentional shifts and is thought to play a decisive role in visual stability. Despite this importance, the nature of the memory underlying priming remains controversial. To understand more fully the necessary conditions for priming, we contrasted passive versus active viewing of visual search arrays. There was no priming from passive viewing of search arrays, while it was strong for active search of the same displays. Displays requiring no search resulted in no priming, again showing that search is needed for priming to occur. Finally, we introduced working memory load during visual search in an effort to disrupt priming. The memorized items had either the same colors as or different colors from the visual search items. Retaining items in working memory inhibited priming of the working memory task-relevant colors, while little interference was observed for unrelated colors. The picture that emerges of priming is that it requires active attentional processing of the search items in addition to the operation of visual working memory, where the task relevance of the working memory load plays a key role.
\end{abstract}

\footnotetext{
Á. Kristjánsson $(\varangle)$

Faculty of Psychology, University of Iceland,

Reykjavík, Iceland

e-mail: ak@hi.is

Á. Kristjánsson · J. Driver

Institute of Cognitive Neuroscience, University College London, London, UK

\section{S. Saevarsson}

Neuropsychology Research Group (EKN), Bogenhausen

University Hospital, Munich, Germany

J. Driver

Wellcome Department of Neuroimaging, University College

London, London, UK

Keywords Vision · Attention · Priming · Visual working-memory

\section{Introduction}

Priming of visual search has a strong effect upon attentional function (e.g., Maljkovic \& Nakayama, 1994; Sigurdardóttir, Kristjánsson, \& Driver, 2008; for reviews, see Kristjánsson \& Campana, 2010; Lamy, Yashar, \& Rudeman, 2010) and plays a key role in visual exploration (Brascamp, Blake, \& Kristjánsson, 2011). Priming is strong enough to account for large portions of effects traditionally attributed to explicit, goal-directed top-down control in search (Kristjánsson, Wang, \& Nakayama, 2002; Wang, Kristjánsson, \& Nakayama, 2005; Wolfe, Butcher, Lee, \& Hyle, 2003) and has been thought to reflect facilitated attention shifts (e.g., Becker, 2008; Brascamp et al., 2011; Kristjánsson, 2008; Lamy et al., 2010). Any role of higher-level effects such as priming of response selection or facilitated episodic memory is relatively small (Ásgeirsson \& Kristjánsson, 2011; Lamy, Zivony, \& Yashar, 2011; but see Huang, Holcombe, \& Pashler, 2004). No study has yet conclusively addressed the minimum requirements for priming or the question of how much secondary load priming tolerates. Our aim was to identify such boundary conditions.

Maljkovic and Nakayama (1994) argued that pop-out of a singleton was primed between trials. If pop-out is primed, many accounts of visual attention (Theeuwes, 2010; Treisman, 1988; Wolfe, 1994) predict that even with passive viewing, priming should occur, since pop-out is assumed to be automatic and bottom-up. Brascamp et al. (2011) compared priming from pop-out trials and choice trials with only two items on the screen. Priming from attentional choice between two differently colored items was as strong as that from popout trials, indicating that priming is driven by choice, not pop- 
out. Inspired by this attentional-choice account of priming, in Experiment 1A, we attempted to uncover the processing requirements for priming by contrasting passive viewing and active search of identical visual search arrays. If attentional choice is required for priming, we should expect priming only in the active-search condition. Experiment 1B tested priming from displays not requiring search.

Working memory (WM) is thought to keep track of information essential for ongoing behavior. Awh and Jonides (2001) argued that WM and attention share function and neural resources. Spatial rehearsal engages top-down processes modulating processing at early stages of visual analysis (Awh, Jonides, \& Reuter-Lorenz, 1998; see also de Fockert, Rees, Frith, \& Lavie, 2001), and memorizing a visual feature leads to an attentional bias toward that feature (e.g., Downing, 2000; Olivers, Meijer, \& Theeuwes, 2006; Soto, Heinke, Humphreys, \& Blanco, 2005). Active maintenance of information in WM may be accomplished through automatic (Hasher \& Zacks, 1979; Soto \& Humphreys, 2008) focal shifts of spatial attention to memorized features or locations (Olivers, Peter, Houtkamp, \& Roelfsema, 2011). If priming reflects facilitated attentional choice, a concurrent WM task should then disrupt the priming. Surprisingly, Lee, Mozer, and Vecera (2009) observed no interference from a WM task upon priming. But they may not have performed a sufficiently stringent test of WM dependence of priming, since they showed only that unrelated feature values in WM minimally affected priming. Task relevance of such WM load was not addressed. Task relevance and feature similarity affect priming (Kristjánsson, 2006, 2009), and in Experiment 2, we therefore tested effects of task-relevant, or -irrelevant, WM load upon priming.

\section{Experiment 1A}

To identify the minimum processing required for priming to occur, we measured the differences between priming from a searched array of items and priming from a passively viewed one. If priming reflects facilitated bottom-up pop-out, priming should occur independently of whether a task is performed. But if the effect involves priming of attentional choice (Brascamp et al., 2011), priming should require active search.

\section{Method}

Observers viewed displays containing either a red target diamond among green distractors or vice versa and, on different blocks, blue targets among yellow distractors (see Fig. 1a). The two possible search sets were never intermingled within blocks. A large black or white annulus appeared 800-1,400 ms (randomly determined) before each trial, indicating whether observers were supposed to perform the search and report the notch location on the target diamond on that trial (see Fig. 1a). Which annulus color denoted which task was counterbalanced across observers. Whether the task was active or passive on a given trial was determined randomly (probability $=.5$ ). The observers therefore had to actively monitor the display throughout, since task requirements were unpredictable.

\section{Observers}

Six participants with normal or corrected-to-normal visual acuity (23-39 years of age; 3 female) participated in 4,000 trials, run in blocks of 200 trials.

\section{Equipment}

The experimental displays were programmed in $\mathrm{C}$ and presented on a $75-\mathrm{Hz}$ CRT.

\section{Stimuli and procedure}

Each trial started with the presentation of a black $(0.8$ $\left.\mathrm{cdm}^{-2}\right)$ or white $\left(56.6 \mathrm{cdm}^{-2}\right)$ fixation cross and a similarly colored annulus for $800-1,400 \mathrm{~ms}$ on a mid-gray background $\left(15.8 \mathrm{cdm}^{-2}\right)$, followed by a search display containing three diamond shapes (each sized $2.4^{\circ} \times 2.4^{\circ}$, at $4.05^{\circ}$ from screen center; see Fig. 1a) on the same background (the annuli remained on the screen). The diamonds were always at maximum distance from each other on the imaginary circle. On half the trials, the singleton was either a red $\left(22.7 \mathrm{cdm}^{-2}\right)$ target among two green distractors $(27.6$ $\mathrm{cdm}^{-2}$ ) or vice versa. On the other half, the singleton was a blue $\left(24.6 \mathrm{cdm}^{-2}\right)$ target diamond among yellow (37.4 $\mathrm{cdm}^{-2}$ ) distractors (or vice versa). On active trials (50\%), observers were instructed to search for the oddly colored diamond and judge (by keypress) whether it had a 15' notch at the top or the bottom (see Kristjánsson, Sigurjónsdóttir, \& Driver, 2010). On passive trials, observers viewed the display and pressed a key when it self-terminated. The presentation time for each observer was a running average of their preceding response times (RTs) on active trials (updated online after each active trial) to equate viewing time between conditions $(1,000 \mathrm{~ms}$ on the first trial).

\section{Results}

Figure 1b shows RTs on active trials as a function of whether the preceding trials were passive or active. The data points for active and passive denote identical conditions, differing only in whether passive or active displays preceded them. The average RT on passive trials (not shown in the figure) was $1,158 \mathrm{~ms}$ and did not show any modulation by 
Fig. 1 Design and results of Experiments $1 \mathrm{~A}$ and 1B. a Sequence of events in Experiment 1A. The different brightness of the annuli (presented 800-1,400 ms before the search display) denoted which task the observers were to perform (passive or active; role of black and white was counterbalanced across observers). The observers then either responded by keypress whether the oddly colored diamond had a cutoff at the top or bottom (active trials) or passively viewed the displays (passive trials). b Performance on active trials of the search task depended on whether, on the previous trials, the observers had actively searched the arrays (active; white symbols) or had viewed them passively (passive; black symbols). So, for example, the white symbols for 2 on the abscissa denote performance on an active trial with two preceding active trials of the same target color, and black for 2 on the abscissa denotes an active trial with two passive trials with the same target color. c Priming effects between adjacent active trials (light gray) versus active trials preceded by passive trials (dark gray) when the colors were similar. d Design of Experiment 1B. e Results of Experiment 1B a
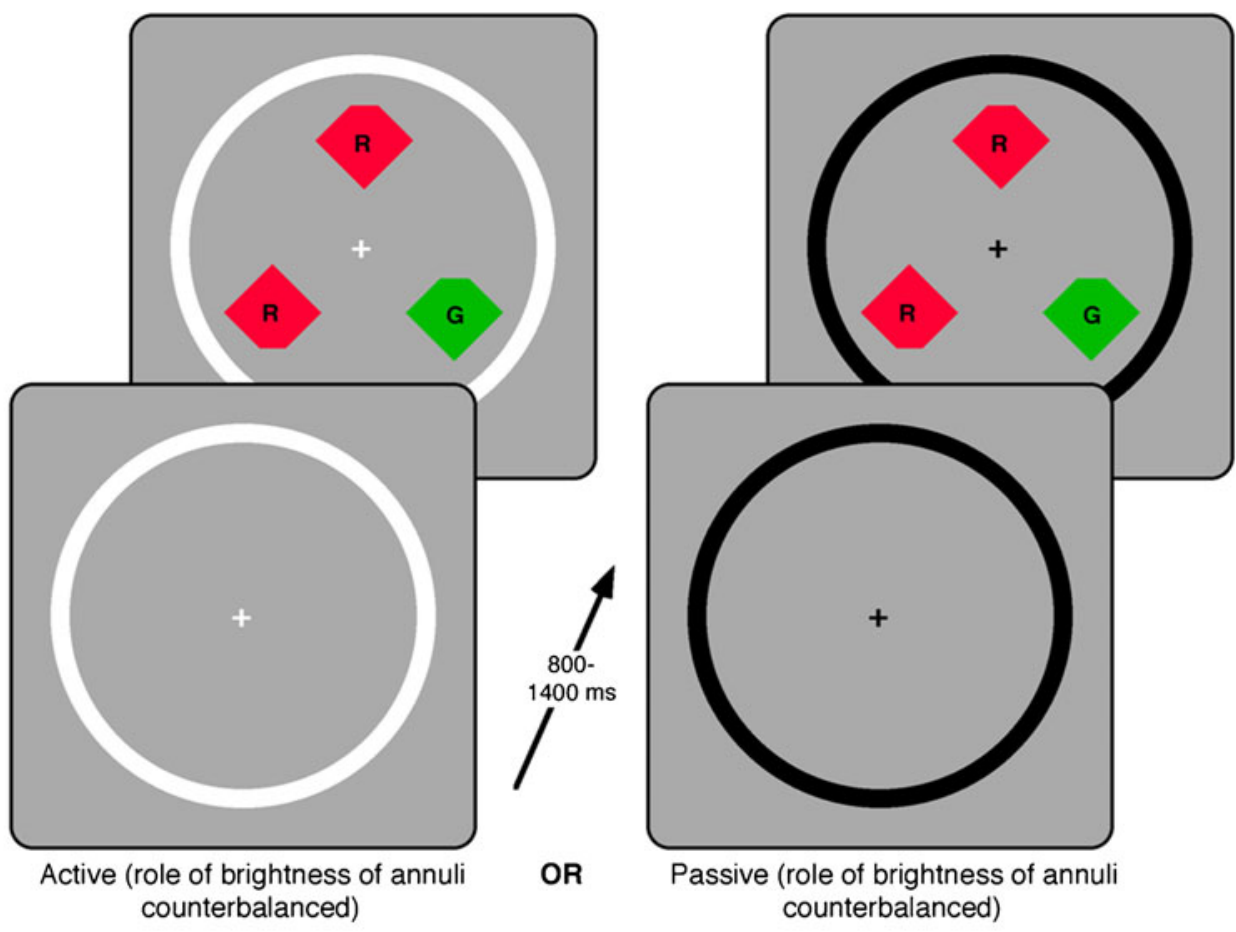

b

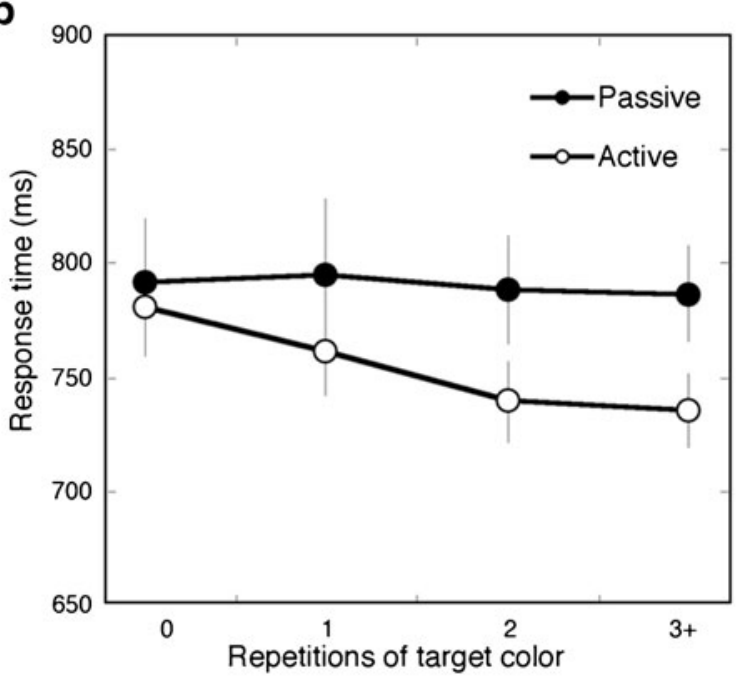

d
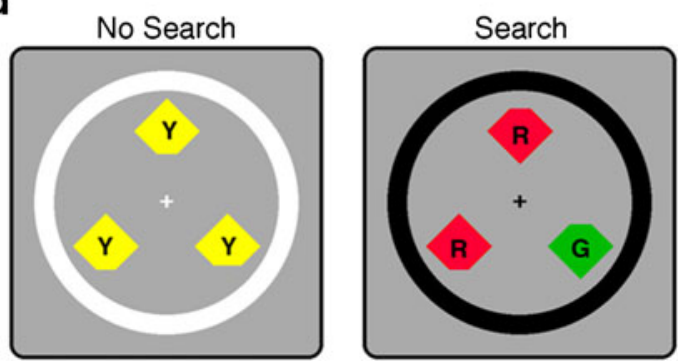

C
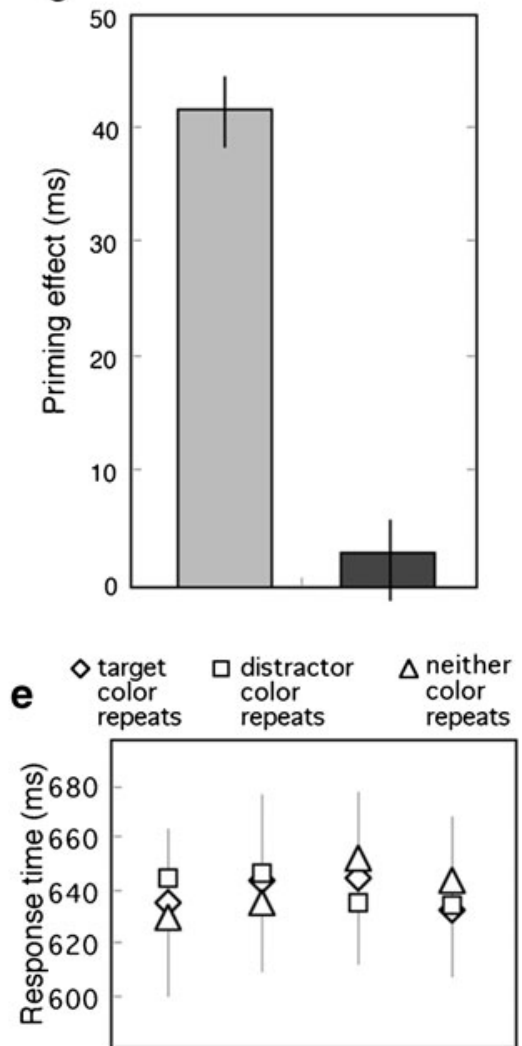

repetition $(p s>4)$. There was no priming from preceding passively viewed displays (black disks), while the priming was strong from preceding active trials (white disks). A 2 (passive or active preceding trials) $\times 4$ (repetition) repeated measures ANOVA tested the interaction between repetition and test. The main effect of repetition was significant, $F(3$, $15)=3.90, p=.03$. The main effect of task (active/passive) was not quite significant, $F(1,5)=3.67, p=.113$, but most 
important, the interaction between the two factors was significant, $F(3,15)=4.50, p=.019$, confirming the difference in priming patterns between conditions. Figure 1c further confirms this by plotting strong priming effects for adjacent active trials but nonexistent priming for active trials preceded by passive trials. There was no difference in performance or priming for the different search types (red and green vs. blue and yellow), $F<1, p>.6$.

\section{Discussion}

Experiment 1 assessed minimum task requirements for priming of pop-out. Pop-out has been thought to be a bottom-up automatic process (Theeuwes, 2010; Treisman, 1988). It should therefore be hard for observers not to notice that the target and pop-out should occur even with passive viewing. Our results indicate that priming is critically dependent upon identifying the target. Priming is not of effortless, automatic pop-out. Instead, the theoretical implications are consistent with the findings of Brascamp et al. (2011), who showed that PoP reflects priming of attentional choice (see also Goolsby \& Suzuki, 2001; Yashar \& Lamy, 2010). Active identification of the target stimulus is required for priming.

\section{Experiment 1B}

Before firm conclusions can be drawn, we may ask, how passive was the passive condition? Observers may simply have ignored the display, closed their eyes, or turned their gaze away. Another possibility is that they actively suppressed pop-out effects, since they were told not to perform the task. Although the former possibility is unlikely, since if this caused no priming, observers would have to do this on every trial, the second possibility cannot easily be ruled out without additional data. Second, we have not ruled out low-level color priming from repeated passive displays, potentially obscured because the search displays contained both target and distractor colors.

Experiment $1 \mathrm{~B}$ therefore serves to further bolster our conclusions from Experiment 1A. We tested any priming from trials with three identical diamonds requiring no search but involving discrimination. If priming truly requires search, we should not see priming from nonsearch displays. Second, if no priming is seen, active suppression of pop-out can be ruled out, since the display must be viewed and responded to.

\section{Method}

During the no-search trials, all diamonds had the same color and a notch in the same location (see Fig. 1d). The observers indicated whether the notch was at the right, left, top, or bottom. On every fifth trial (on average), a search display was presented where the observers located the odd-one-out and determined the location of the notch (location determined randomly on each trial). We tested conditions where the no-search color (say, yellow) was the target color on the search trial (e.g., yellow target among green distractors), was the distractor color on the search trial, or was unrelated to search (e.g., red target among green distractors). Again, the brightness of the large annuli distinguished between the two tasks (counterbalanced). Four observers participated in 2,400 trials in 12 blocks.

\section{Results}

There was no evidence of any priming from the nonsearch notch discrimination. Figure 1e shows performance on a search trial, depending on how many nonsearch trials preceded it, with different symbols denoting whether the nosearch color was the target color, was the distractor color, or did not appear in the search display. A 3 (condition) $\times 4$ (repetition) repeated measures ANOVA confirmed no main effect of either condition, $F(2,6)=0.82, p=.48$, or repetition, $F(3,9)=0.63, p=.61$, nor was there an interaction between the factors, $F(6,18)=1.15, p=.37$. These findings bolster the argument that search is necessary for priming and also rule out any low-level color priming effects.

\section{Experiment 2}

Experiment 2 addressed the question of what is needed to disrupt priming, measuring the effects of task-relevant and irrelevant WM load upon priming. As in Experiments 1A and $1 \mathrm{~B}$, the annulus color denoted the task: to either memorize items and report on whether a change occurred in a subsequent display or locate the notch on the odd-diamondout. The memory task contained either similarly or differently colored diamonds to the visual search items (again red vs. green and blue vs. yellow, independently contrasted). When black denoted the WM task, observers had to memorize the display on the first trial with a black annulus and, for the next black annulus trial, report whether a change had occurred. On every trial with a white annulus, they performed visual search. For the other half of the observers, the roles of the annuli colors were reversed.

\section{Method}

The task-denoting annulus and fixation cross appeared 800$1,400 \mathrm{~ms}$ before the search array. When the annulus denoting the memory task appeared, the observers memorized the colors and locations of the three diamonds. On the next instance of that annulus color, they pressed a key according to whether the display had changed or not. The next 
presentation of a display with that annulus denoted a display to be remembered, and so on (see Fig. 2a). On different blocks of 200 trials, the search was either for a red target among green distractors, or vice versa, or for a blue target among yellow distractors, or vice versa. The WM items were either red/green or blue/yellow, to contrast relevant versus irrelevant colors within a block. Twenty-five percent of the trials within a block were memory and test trials, while the rest were visual search trials (trial type determined randomly for each trial). Three kinds of change could occur (on $50 \%$ of memory test trials): (1) change in location of pop-out target (color unchanged), (2) change in pop-out target color (location unchanged), and (3) change in both color and position. Observers did not distinguish different
Fig. 2 Design and results of Experiment 2. a The task in experiment 2. Observers were supposed to either memorize the three diamonds or find the odd-diamond out and report the location of the notch (up-ordown), depending on the brightness of the surrounding annulus. In the figure, white denotes the working memory (WM) task, while black denotes visual search (the roles of the colors were counterbalanced across observers). The figure shows the case where the search colors were irrelevant to the WM task. b Response times by repetition as a function of relevance of search task to WM task and of whether the WM task was performed correctly or not. c Correlations for individual observers between size of priming effects and WM performance (measured with $A^{\prime}$ ) for task-relevant versus taskirrelevant colors

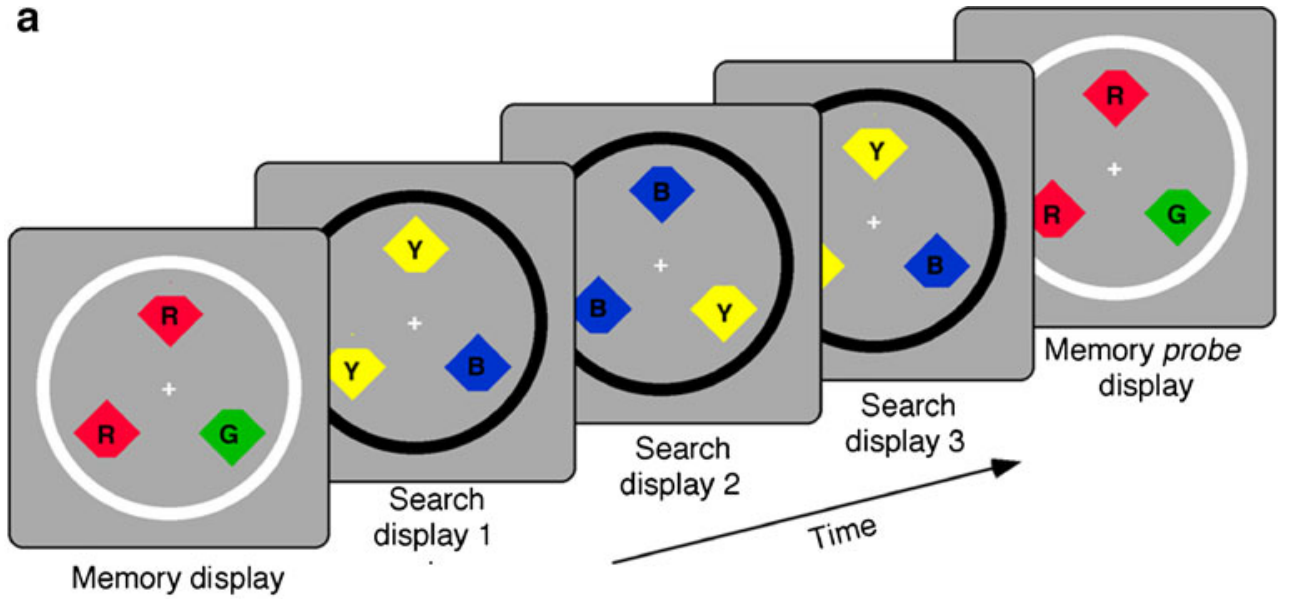

b

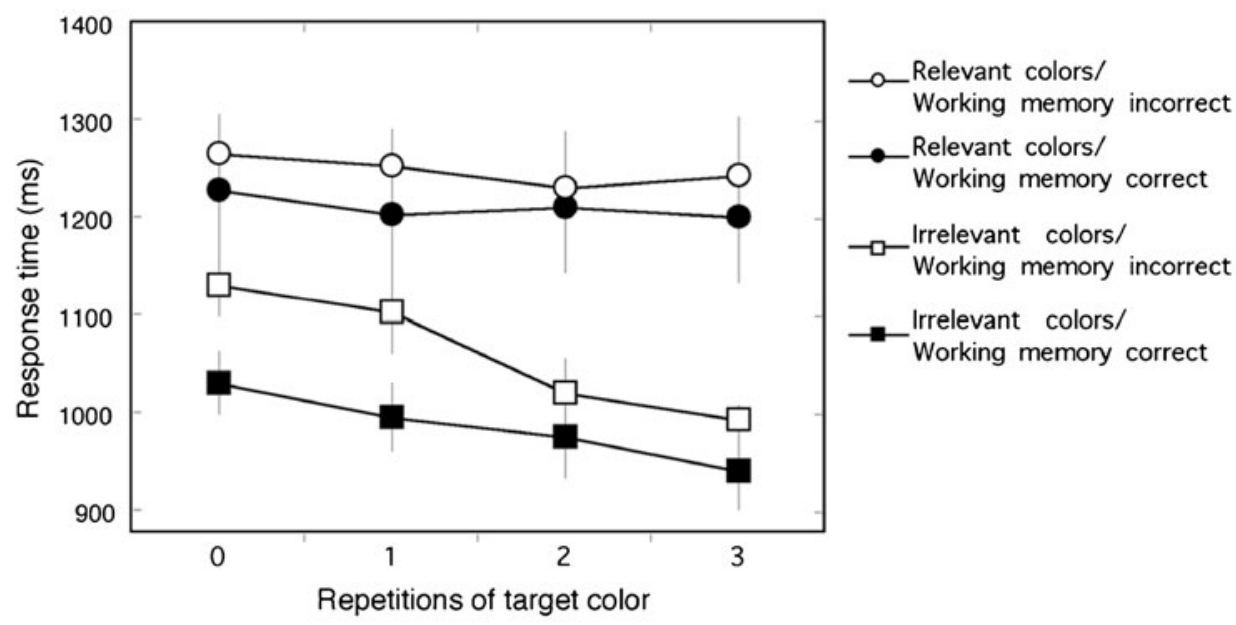

C

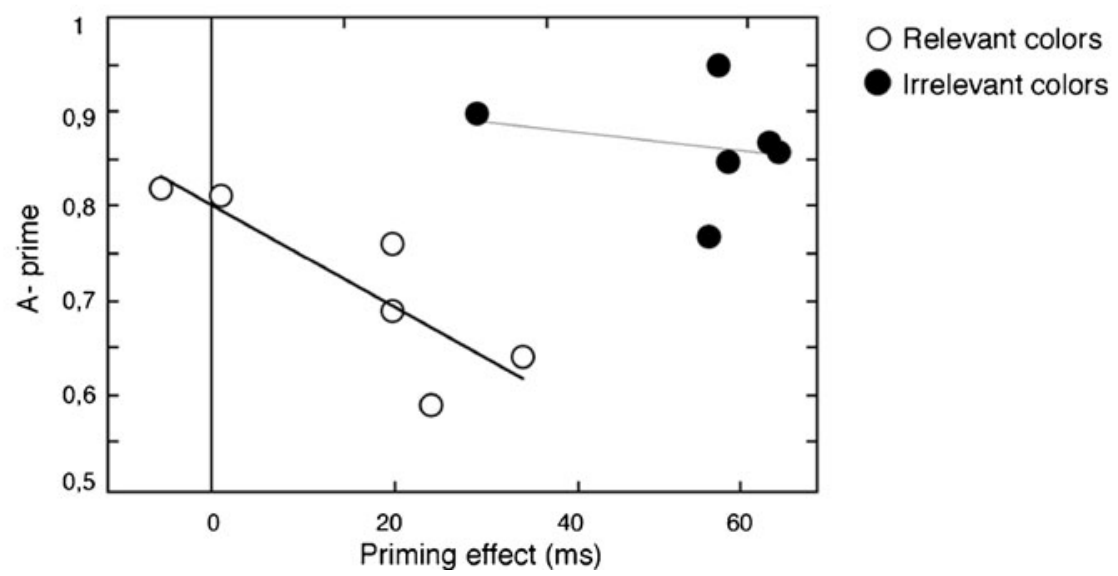


changes but only reported whether any change had occurred. To increase the number (above chance) of consecutive repetitions of target color in the visual search, the probability of target-color repetition was determined with the function $100-(20 * \sqrt{N})$, where $N$ denotes the number of previous presentations of the same target color. Other details of experimental methods were comparable to those in the previous experiments, and the same 6 observers as in Experiment 1A participated in 4,000 trials in blocks of 200 .

\section{Results}

Figure $2 \mathrm{~b}$ shows priming for search trials as a function of whether the WM task was performed correctly and whether the colors to be remembered were the same as during visual search (relevant vs. irrelevant). There was very little difference depending on the three change conditions (color change, $78.3 \%$; position change, $80.3 \%$; change in both, $80.1 \%$ ). The results show that there was little or no priming from repetition of the WM relevant colors, while strong priming was observed from irrelevant colors. If observers were, for example, required to remember red and green diamonds, priming was robust for search for blue versus yellow, but not for search for red versus green. This was confirmed in a 2 (relevant vs. irrelevant colors) $\times 2$ (performance on WM task) $\times 4$ (repetition) repeated measures ANOVA. The main effect of relevance was significant, $F(1$, $5)=15.8, p=.011$, as were the main effects of performance, $F$ $(1,5)=7.21, p=.044$, and repetition, $F(3,15)=4.26, p=.023$. Only the most important interaction was significant, that between repetition and relevance, $F(3,15)=3.489, p=.043$. Other interactions were not significant $(p s>.4)$, which most notably shows that the priming effects were similar irrespective of whether WM performance was correct or not. Error rates during search were $4.3 \%$ overall. Figure $2 \mathrm{c}$ shows priming effects for individual observers as a function of WM performance $^{1}$ for the task-relevant (white symbols) and -irrelevant (black symbols) colors, depending on priming magnitude (RT difference between the first and fourth or more color presentations). Better WM performance correlated negatively and significantly, $r=.86, p=.024$, with the size of priming effects for the task-relevant colors (white symbols in Fig. 2c) but not for the task-irrelevant ones, $r=.334, p=.45$ (black symbols in Fig. 2c). Finally, a $t$-test confirmed that $A^{\prime}$ varied significantly as a function of task relevance, $t(5)=2.89, p=.0178$.

${ }^{1}$ This was measured with the $A^{\prime}$ measure of changes in sensitivity:

$A^{\prime}=0.5+\left[\frac{(H-g)(1+H-g)}{4 H(1-g)}\right]$,

where $H$ denotes hit rate and $g$ denotes false alarm rate (see, e.g., Donaldsson, 1993; Grier, 1971).

\section{Discussion}

The results of Experiment 2 show that task-relevant memory load strongly disrupts priming. The evidence for disruption from the irrelevant colors is, on the other hand, meager, for both RT and $A^{\prime}$. Furthermore, for individual observers, WM performance correlated negatively with priming effects only if the colors of the search task were WM relevant, showing interdependence of priming and WM for overlapping colors.

\section{General discussion}

From our effort to identify the boundary conditions for priming of attentional choice (often referred to as PoP), we draw the following conclusions.

1. Priming requires active search. Passive viewing of popout displays does not lead to priming, consistent with the findings of Brascamp et al. (2011), who showed that PoP reflects active attentional choice.

2. WM load can severely disrupt priming when the items to be memorized contain the search features. For taskirrelevant items, the disruption is minor.

3. Priming reflects the operation of attentional mechanisms whose function overlaps with visual WM. Here, task relevance plays a major role.

Priming has been shown to play a major role in visual exploration (Brascamp et al., 2011; Kristjánsson \& Campana, 2010), allowing facilitated reorientation to taskrelevant items (Ballard et al., 1992; Maljkovic \& Nakayama, 1994), and to affect figure-ground assignment in ambiguous displays (Kristjánsson, 2009). Our experiments involve the first attempt to uncover the necessary conditions for priming. The finding that WM load influences primed attention shifts lends support to proposals that WM and attention share similar neural mechanisms, such as the well-known parietal-frontal circuits that play a key role in visual attention and maintenance of representations of items held in WM (Awh \& Jonides, 2001). This is consistent with studies of neural correlates of priming (from fMRI, Geng et al., 2006; Kristjánsson, Vuilleumier, Schwartz, Macaluso, \& Driver, 2007; and Rorden, Kristjánsson, Pirog-Revill, \& Saevarsson, 2011; TMS, Campana, Cowey, \& Walsh, 2002; and O'Shea, Muggleton, Cowey, \& Walsh, 2007; neuropsychology, Kristjansson, Vuilleumier, Malhotra, Husain, \& Driver, 2005, and Saevarsson, Jóelsdóttir, Hjaltason, \& Kristjánsson, 2008; combined TMS and ERP, Taylor, Muggleton, Kalla, Walsh, \& Eimer, 2011), which show that attentional mechanisms are strongly involved in priming. Our results are also consistent with the suggestion that priming and WM share functional and neural resources (Soto, Llewelyn, \& Silvanto, 2012). Finally, the fact that 
Lee et al. (2009) did not observe disruptive effects of WM load upon priming are still, on the surface, consistent with our findings, since the task relevance of memory load was not tested in their experiments.

\section{Conclusions}

The dramatic effect that intertrial priming has upon visual performance makes it all the more important to understand its characteristics and mechanisms. We have identified minimum requirements for priming and the maximum interference that priming survives, which may form its boundary conditions. Priming does not occur passively but requires attentional choice and reflects the operation of short-term memory mechanisms where task relevance plays a key role.

Acknowledgments A.K. was supported by the research fund of the University of Iceland, and J.D. by the Wellcome Trust and a Royal Society Anniversary Research Professorship. We are extremely grateful to Dr. J. Hopfinger and three anonymous referees for comments upon previous versions of the manuscript. Jon Driver passed away during the preparation of this manuscript. A.K. is forever grateful for his collaboration on this study and other projects over the last decade.

\section{References}

Ásgeirsson, Á. G., \& Kristjánsson, Á. (2011). Episodic retrieval and feature facilitation in intertrial priming of visual search. Attention, Perception, \& Psychophysics, 73, 1350-1360.

Awh, E., \& Jonides, J. (2001). Overlapping mechanisms of attention and working memory. Trends in Cognitive Sciences, 5, $119-126$.

Awh, E., Jonides, J., \& Reuter-Lorenz, P. A. (1998). Rehearsal in spatial working memory. Journal of Experimental Psychology. Human Perception and Performance, 24, 780-790.

Ballard, D. H., Hayhoe, M. M., Li, F., Whitehead, S. D., Frisby, J. P., Taylor, J. P., \& Fisher, R. B. (1992). Hand-eye coordination during sequential tasks. Philosophical Transactions of the Royal Society: London B, 337, 331-339.

Becker, S. I. (2008). The mechanism of priming: Episodic retrieval or priming of pop-out? Acta Psychologica, 127, 324-339.

Brascamp, J. W., Blake, R., \& Kristjánsson, Á. (2011). Deciding where to attend: Priming of pop-out drives target selection. Journal of Experimental Psychology. Human Perception and Performance, 37, 1700-1707.

Campana, G., Cowey, A., \& Walsh, V. (2002). Priming of motion direction and area V5/MT: A test of perceptual memory. Cerebral Cortex, 12, 663-669.

de Fockert, J. W., Rees, G., Frith, C. D., \& Lavie, N. (2001). The role of working memory in visual selective attention. Science, 291, $1803-1806$.

Donaldson, W. (1993). Accuracy of d' and A' as estimates of sensitivity. Bulletin of the Psychonomic Society, 31, 271-274.

Downing, P. E. (2000). Interactions between visual working memory and selective attention. Psychological Science, 11, 467-473.

Geng, J. J., Eger, E., Ruff, C., Kristjánsson, Á., Rothsthein, P., \& Driver, J. (2006). On- line attentional selection from competing stimuli in opposite visual fields: Effects on human visual cortex and control processes. Journal of Neurophysiology, 96, 2601-2612.
Grier, J. B. (1971). Nonparametric indexes for sensitivity and bias: Computing formulas. Psychological Bulletin, 75, 424-429.

Goolsby, B. A., \& Suzuki, S. (2001). Understanding priming of colorsingleton search: Roles of attention at encoding and retrieval. Perception \& Psychophysics, 63, 929-944.

Hasher, L., \& Zacks, R. T. (1979). Automatic and effortful processes in memory. Journal of Experimental Psychology. General, 108, 356-388.

Huang, L., Holcombe, A. O., \& Pashler, H. (2004). Repetition priming in visual search: Episodic retrieval, not feature priming. Memory \& Cognition, 32, 12-20.

Kristjánsson, Á. (2006). Simultaneous priming along multiple dimensions in visual search task. Vision Research, 46, 2554-2570.

Kristjánsson, Á. (2008). "I know what you did on the last trial" - a selective review of research on priming in visual search. Frontiers in Bioscience, 13, 1171-1181.

Kristjánsson, Á. (2009). Learning in shifts of transient attention improves recognition of parts of ambiguous figure-ground displays. Journal of Vision, 9(21), 1-11.

Kristjánsson, Á., \& Campana, G. (2010). Where perception meets memory: A review of priming in visual search. Attention, Perception, \& Psychophysics, 72, 5-18.

Kristjánsson, Á., Sigurjónsdóttir, Ó., \& Driver, J. (2010). Fortune and reversals of fortune in visual search: Reward contingencies for pop-out targets affect search efficiency and target repetition effects. Attention, Perception \& Psychophysics, 72, 1229-1236.

Kristjánsson, Á., Vuilleumier, P., Malhotra, P., Husain, M., \& Driver, J. (2005). Priming of color and position during visual search in unilateral spatial neglect. Journal of Cognitive Neuroscience, 17, 859-873.

Kristjánsson, Á., Vuilleumier, P., Schwartz, S., Macaluso, E., \& Driver, J. (2007). Neural basis for priming of pop-out revealed with fMRI. Cerebral Cortex, 17, 1612-1624.

Kristjánsson, Á., Wang, D., \& Nakayama, K. (2002). The role of priming in conjunctive visual search. Cognition, 85, 37-52.

Lamy, D., Yashar, A., \& Ruderman, L. (2010). A dual-stage account of inter-trial priming effects. Vision Research, 48, 1274-1279.

Lamy, D., Zivony, A., \& Yashar, A. (2011). The role of search difficulty in intertrial feature priming. Vision Research, 51, 2099-2109.

Lee, H., Mozer, M. C., \& Vecera, S. P. (2009). Mechanisms of priming of pop-out: Stored representations or feature-gain modulations? Attention, Perception, \& Psychophysics, 71, 1059-1071.

Maljkovic, V., \& Nakayama, K. (1994). Priming of pop-out: I. Role of features. Memory \& Cognition, 22, 657-672.

O'Shea, J., Muggleton, N. G., Cowey, A., \& Walsh, V. (2007). Human frontal eye fields and spatial priming of pop-out. Journal of Cognitive Neuroscience, 19, 1140-1151.

Olivers, C. N. L., Meijer, F., \& Theeuwes, J. (2006). Feature-based memory-driven attentional capture: Visual working memory content affects visual attention. Journal of Experimental Psychology. Human Perception and Performance, 32, 1243-1265.

Olivers, C. N. L., Peter, J., Houtkamp, R., \& Roelfsema, P. R. (2011). Different states in visual working memory: When it guides attention and when it does not. Trends in Cognitive Sciences, 15, 327-334.

Rorden, C., Kristjánsson, Á., Pirog-Revill, K., \& Saevarsson, S. (2011). Neural correlates of inter-trial priming and role-reversal in visual search. Frontiers in Human Neuroscience, 5, 151.

Saevarsson, S., Jóelsdóttir, S., Hjaltason, H., \& Kristjánsson, Á. (2008). Repetition of distractor sets improves visual search performance in hemispatial neglect. Neuropsychologia, 45, 1161-1169.

Sigurdardóttir, H. M., Kristjánsson, Á., \& Driver, J. (2008). Repetition streaks increase perceptual sensitivity in brief visual search displays. Visual Cognition, 16, 643-658.

Soto, D., Heinke, D., Humphreys, G. W., \& Blanco, M. J. (2005). Early, involuntary top-down guidance of attention from working memory. Journal of Experimental Psychology. Human Perception and Performance, 31, 248-261. 
Soto, D., \& Humphreys, G. W. (2008). Stressing the mind: The effect of cognitive load and articulatory suppression on attentional guidance from working memory. Attention, Perception, \& Psychophysics, 70, 924-934.

Soto, D., Llewelyn, D., \& Silvanto, J. (2012). Distinct causal mechanisms of attentional guidance by working memory and repetition priming in early visual cortex. Journal of Neuroscience, 32, 3447-3452.

Taylor, P. C. J., Muggleton, N. G., Kalla, R., Walsh, V., \& Eimer, M. (2011). TMS of the right angular gyrus modulates priming of popout in visual search: Combined TMS-ERP evidence. Journal of Neurophysiology, 106, 3001-3009.

Theeuwes, J. (2010). Top-down and bottom-up control of visual selection. Acta Psychologica, 123, 77-99.
Treisman, A. (1988). Features and objects: The fourteenth Bartlett memorial lecture. Quarterly Journal of Experimental Psychology. A, Human Experimental Psychology, 40A, 201-237.

Wang, D., Kristjánsson, Á., \& Nakayama, K. (2005). Efficient visual search without top-down or bottom-up guidance. Perception \& Psychophysics, 67, 239-253.

Wolfe, J. M. (1994). Guided Search 2.0: A revised model of visual search. Psychonomic Bulletin \& Review, 1, 202-238.

Wolfe, J. M., Butcher, S. J., Lee, C., \& Hyle, M. (2003). Changing your mind: On the contributions of top-down and bottom-up guidance in visual search for feature singletons. Journal of Experimental Psychology. Human Perception and Performance, 29, 483-502.

Yashar, A. \& Lamy, D. (2010). Intertrial Repetition Affects Perception: The Role of Focused Attention. Journal of Vision, 10, 14, 3, $1-8$. 